\title{
Rechtsgeschichte
}

www.rg.mpg.de

http://www.rg-rechtsgeschichte.de/rg16

Zitiervorschlag: Rechtsgeschichte Rg 16 (2010)

$\operatorname{Rg} \mathbf{1 6} 2010$

$288-292$

http://dx.doi.org/10.12946/rg16/288-292

\section{Juan B. Cañizares Navarro}

\section{Cochabamba, Bolivien, Januar 1789 - August 1791}

Ein Beitrag zu bildlichen Darstellungen unmenschlicher und entwürdigender Behandlungen 


\section{Cochabamba, Bolivien, Januar I789-August I79I}

Ein Beitrag zu bildlichen Darstellungen unmenschlicher und entwürdigender Behandlungen*

Die bildlichen Darstellungen in diesem Band der Rechtsgeschichte sind Teil eines Werkes, das unser Wissen über die Amtsführung der Verwaltungsorgane von Santa Cruz de la Sierra und die Justizpraxis der dortigen Beamten gegen Ende der Kolonialzeit wesentlich bereichert. Es handelt sich um die Edition einer Gerichtsakte, bestehend aus einer Berufungsschrift und einigen Abbildungen. Durch diese wollte der Privatmann Judas Tadeo die Darlegungen seiner Berufung gegen die Umstände seiner Gefangenschaft in Cochabamba stützen und die während seiner dortigen Haft erlittenen Übergriffe belegen. Ergänzt wird die Ausgabe durch Studien dreier Wissenschaftler, die vom Standpunkt ihrer jeweiligen Disziplin die verschiedenen Fragen beantworten, welche die Multidisziplinarität des kleinen Werkes aufwirft. Die Abfolge der veröffentlichten Bilder stellt mit bemerkenswerter Präzision die sowohl direkt als auch indirekt am Gerichtsprozess beteiligten Personen und die Orte dar, an denen die Diffamierung des Judas Tadeo stattfindet. Laut Blatt eins der Gerichtsakte liegen der Berufung insgesamt acht Abbildungen bei; die dritte Abbildung war jedoch in Boliviens Archivo y Biblioteca Nacionales, aus denen die Gerichtsakte stammt, nicht auffindbar.

Nach einer kurzen Zusammenfassung des Inhalts der Illustrationen wird sich dieser kurze Beitrag mit der Behandlung der durch die Abbildungen aufgeworfenen Fragestellungen durch die drei Fachleute beschäftigen und mit einem kritischen Urteil über ihre Beiträge schließen; $\mathrm{da}$ es sich vor allem um eine Marginalie zu den
Illustrationen handelt, wird auf den Nachweis von Sekundärliteratur verzichtet.

\section{Der Anlass}

Die ersten beiden Zeichnungen zeigen den von Judas Tadeo angegebenen Grund für seine Inhaftierung und seine physische sowie psychische Misshandlung: den vermeintlichen Tod eines seiner Lehrlinge infolge von Peitschenhieben, die Tadeo ausgeteilt hatte. Dabei ist der Lehrling in beiden Abbildungen zu sehen: im ersten Bild erschrocken und auf der Flucht vor Judas' Zurechtweisung, im zweiten von jemandem getragen und augenscheinlich tot. Das ist zumindest die einhellige Ansicht der Fachleute, welche die betreffende Gerichtsakte untersucht haben. Allerdings waren die Peitschenhiebe und der vermeintliche Tod des Lehrlings wohl nicht der einzige Grund für die Inhaftierung Judas Tadeos; hinzu kamen Schulden, die Tadeo bei dem Vermieter der Räume hatte, in denen er sein Gewerbe ausübte, und der zugleich Stadtrichter (alcalde ordinario) von Cochabamba war - so jedenfalls lässt es sich nach Enciso Contreras, der eine der Studien zu den Bildern verfasst hat, der Akte entnehmen.

Dementsprechend stellt sich die Frage, nach welchem Recht die Auspeitschung zu beurteilen war, welches ihre rechtliche Natur war, in welchen Fällen sie angewendet werden durfte und wie. Und wenn sich Judas Tadeo andererseits als Täter eines Tötungsdelikts herausstellte, wäre es dann angebracht gewesen, ihn einzusperren und sein Gut zu beschlagnahmen? Verhielten

Inch Calvimonte Marcela und Marta Irurozqui Victoriano

(Hg.), Justicia y tortura en los

Andes. Recurso de Judas Tadeo

Andrade ante la Audiencia de

Charcas, I79I. Estudios críticos

y transcripción, Madrid: Consejo

Superior de Investigaciones Cien-

tíficas y Archivo y Biblioteca Na-

cionales de Bolivia 2007, I78 f.,

응 ISBN 978-84-00-08568-I 
sich schließlich die Behörden von Cochabamba rechtskonform bei der Art, wie sie gegen Judas Tadeo vorgingen, wenn diese Tatsache wahr war?

Bezüglich dieser Fragen konzentrieren sich die Informationen, die die beiden anderen $\mathrm{Au}-$ toren, Mendoza Loza und Peralta Ruiz, zusammentragen, auf die Praxis der kommentierten Maßnahme auf hispanoamerikanischem Gebiet in der Kolonialzeit. Sie stützen sich jeweils auf die Gerichtsakte und die Historiographie, ohne mehr als die zuvor genannten Details zu dieser Problematik im Hinblick auf den uns beschäftigenden Rechtsfall beizusteuern. Es ist Enciso Contreras, der auf der Grundlage von Sekundärliteratur zur Folter einige Details zur Strafe der Auspeitschung schildert, auf die Entstehungsgeschichte dieser Maßnahme hinweist, auf die Tatbestände, bei denen ihr Einsatz vorgesehen war, auf die Anwendung und die Subjekte der Auspeitschung im Gebiet des Río de la Plata. Contreras wagt auf der Grundlage der Informationen der Gerichtsakte von Judas Tadeo sogar eine These über die verbreitete Anwendung der Auspeitschung in Cochabamba. Sämtliche Informationen, die die Experten gesammelt haben, tragen zu einem vollständigeren Bild der Maßnahme des Auspeitschens bei. Dadurch ermöglichen sie einen Vergleich der Praxis der Auspeitschung zwischen verschiedenen Gebieten Hispanoamerikas und im aufgeklärten Europa, wobei vielleicht die Analyse der in den ersten beiden Abbildungen des Judas Tadeo im Mittelpunkt stehenden Tatbestände ein wenig zu kurz kommt. Denn es gab verschiedene Normen, die sich auf das bezogen, was in diesen zwei Abbildungen dargestellt ist: In den Siete Partidas, in der Nueva Recopilación und in anderen Rechtsnormen wurden die Subjekte, die Fälle, die Form der Anwendung der Auspeitschung als Diszipli- narmaßnahme bei Lehrlingen und die Konsequenzen bei Nichtbeachtung dieser Vorschriften abgehandelt. Ebenso war der Totschlag in diesen Gesetzbüchern nach den bereits genannten Charakteristika ausführlich geregelt. Es gab also Rechtsgrundlagen, die perfekt zu den Umständen in unserem konkreten Fall passten. Behandelte Judas Tadeo seinen Lehrling also korrekt? Und wenn der Ausgepeitschte durch die exzessiven Peitschenhiebe von Judas tatsächlich gestorben war, welche juristischen Konsequenzen waren dann vorgesehen? - Letztendlich ermöglicht der Inhalt dieser Vorschriften zusammen mit den Informationen der Gerichtsakte selbst und der Sekundärliteratur zur Disziplinarmaßnahme der Auspeitschung eine komplette Untersuchung des Gegenstandes der ersten beiden bildlichen Darstellungen und den Vergleich der ausgeführten Praktik in diesem konkreten Fall mit anderen bereits untersuchten Fällen.

\section{Leidensweg im Gefängnis und »Abschied»}

Die Illustrationen vier bis acht beziehen sich auf den Aufenthalt von Judas Tadeo im Gefängnis von Cochabamba. Die Gründe für die Inhaftierung unseres Protagonisten wurden bereits genannt, und in dieser zweiten Gruppe von Bildern können wir die Motive für die Demütigungen kennen lernen, von denen er schriftlich und mit Hilfe seiner Zeichnungen berichtet. Damit werden wir Zeugen der Drangsalierungen, denen er ausgesetzt war, was uns gestattet, uns über die dabei eingesetzten Mittel wie über die daran beteiligten Personen ein genaues Bild zu machen. Die Darstellungen der Bildfolge und der sie begleitende Text reflektieren die Folgen der Inhaftierung von Judas Tadeo für seine Gesundheit, die menschenunwürdige Behandlung 
und sie geben zugleich einen Einblick in die Praxis der Strafverfolgung der Behörden von Cochabamba.

Die vor allem auf Literatur zur Geschichte im La-Plata-Raum und auf Studien zum Gefängniswesen beruhenden Begleittexte konzentrieren sich wiederum auf Ursprung, Zweck und Funktionen des Gefängnisses sowie der als Gefängnis genutzten Orte. Sie diskutieren den Charakter der Haftanstalt als Strafinstitution und liefern wichtige Informationen über ihre architektonischen sowie soziosanitären Charakteristika. Besonders interessant ist der von Enciso Contreras durchgeführte Vergleich der Primärquellen mit dem Forschungsstand hinsichtlich des Ausnahmecharakters oder der Normalität einer Maßnahme wie der im vorliegenden Fall.

Was die gegenüber Judas Tadeo eingesetzten Mittel während seiner Inhaftierung betrifft, kommt die von den Autoren zusammengetragene reiche Forschungsliteratur zu keinem einheitlichen Ergebnis. Es ist die kritische Studie von Enciso Contreras, die das Thema am intensivsten erschließt. Contreras gelingt es, Aussagen zur Folter in Cochabamba, ihrer juristischen Natur, ihrer Grundlage und Daseinsberechtigung ebenso zu treffen wie hinsichtlich der Orte, der Formen und der Instrumente ihrer Durchführung. Allerdings werden die von ihm genannten Quellen im iberischen geographischen Raum nicht mit den entsprechenden Quellen zu Alto Perú verglichen, und er bezieht sich nur wenig auf die konkrete Praxis dieser Zwangsmaßnahmen in Cochabamba, wie es für einen wirklichen Vergleich wünschenswert gewesen wäre. Im Beitrag von Peralta Ruiz ist der kurze bibliographische Überblick über die wichtigsten europäischen Quellen zum Thema Folter und das Ende ihrer Anwendung in Spanien hervorzuheben, wodurch die juristische Natur deutlich wird, welche die zeitgenössischen Autoren, die die Folter befürworteten oder ablehnten, ihr zusprachen.

Abgesehen von diesen Fragen wird in dem besprochenen Werk leider nur in geringem Maße Bezug auf die normativen Quellen zur Folter genommen. So wird auf die Real Cédula verwiesen, mittels derer die Folter in Spanien verboten wurde - ohne dass die Norm erwähnt wird, auf deren Grundlage sie erlaubt gewesen war. Es heißt, die Folter habe "auf einer breiten legalen und juristischen Grundlage " gestanden, ohne diese »legale und juristische Grundlage « zu spezifizieren und mit der in dieser Gerichtsakte beschriebenen durchgeführten Praxis zu vergleichen; es wird behauptet, dass es nicht in allen Strafprozessen in Spanien und im kolonialen Amerika "Folterurteile « gab, ohne diesen Vergleich zu belegen. In dieser Hinsicht wäre die Normgebundenheit der Behörden von Cochabamba zu untersuchen gewesen, da - um nur einige Normen zu nennen, die in den spanischen Kolonien Amerikas zu jener Zeit anwendbar waren - die Siete Partidas dieser Frage einen ganzen Titel widmen und sie in weiteren Titeln thematisiert. Sowohl die Nueva Recopilación als auch die Recopilación de Leyes de los Reynos de las Indias legten den Rechtscharakter der Folter fest, die von der Folter grundsätzlich ausgenommenen Personen, die Voraussetzungen, unter denen sie bei grundsätzlich ausgenommenen oder allen anderen Personen angewendet werden konnte, das Verfahren, die Art ihrer Anwendung oder die Verantwortung des Richters bei der Nichteinhaltung all dieser Regeln.

Folter ist nicht die einzige erniedrigende Maßnahme für Judas Tadeo. Auch die öffentliche Schande - illustriert in den Abbildungen sieben und acht - wird von allen Autoren kommentiert. Sie beziehen sich auf ihren akzessorischen Charakter, da es sich um eine Strafe han- 
delt, die Anwendung findet, bevor Judas Tadeo zur Verurteilung vor die Audiencia de Charcas gebracht wird, und die unter der Aufsicht der Beamten stattfindet, die mit der Ausführung der Strafe beauftragt sind. Um diese Aussagen zu untermauern, stützen sich die Autoren allerdings ausschließlich auf den Inhalt der Gerichtsakte, ohne diese Fragen zu vertiefen. Allein Enciso Contreras informiert über die juristische Natur des Gefängnisses, um zu zeigen, dass es sich nicht um eine Strafe sensu stricto handelte, und kommentiert ausgehend von der Forschungsliteratur zum Strafrecht des Absolutismus, dass öffentliche Schande als Strafe angesehen werden konnte und eine der häufigsten Sanktionsformen war. Wenn er an späterer Stelle von den anderen Strafen spricht, die bei Judas Tadeo Anwendung fanden, führt er ebenfalls aus, dass öffentliche Schande »als entehrende Strafe angesehen " wird, belegt und begründet seine Einschätzung aber nicht. Da wir wenig über die Strafe der öffentlichen Schande wissen, hätte dieser konkrete Fall eine gute Gelegenheit geboten, die Frage etwa nach der Implementierung der Schandstrafe aufzunehmen. Hier hätte das komparative Potential der Quelle ausgeschöpft werden können, auch um die juristische Praxis in der Region von Cochabamba besser kennenzulernen und mit der Praxis in anderen Regionen des kolonialen Amerika vergleichen zu können.

Juan B. Cañizares Navarro 

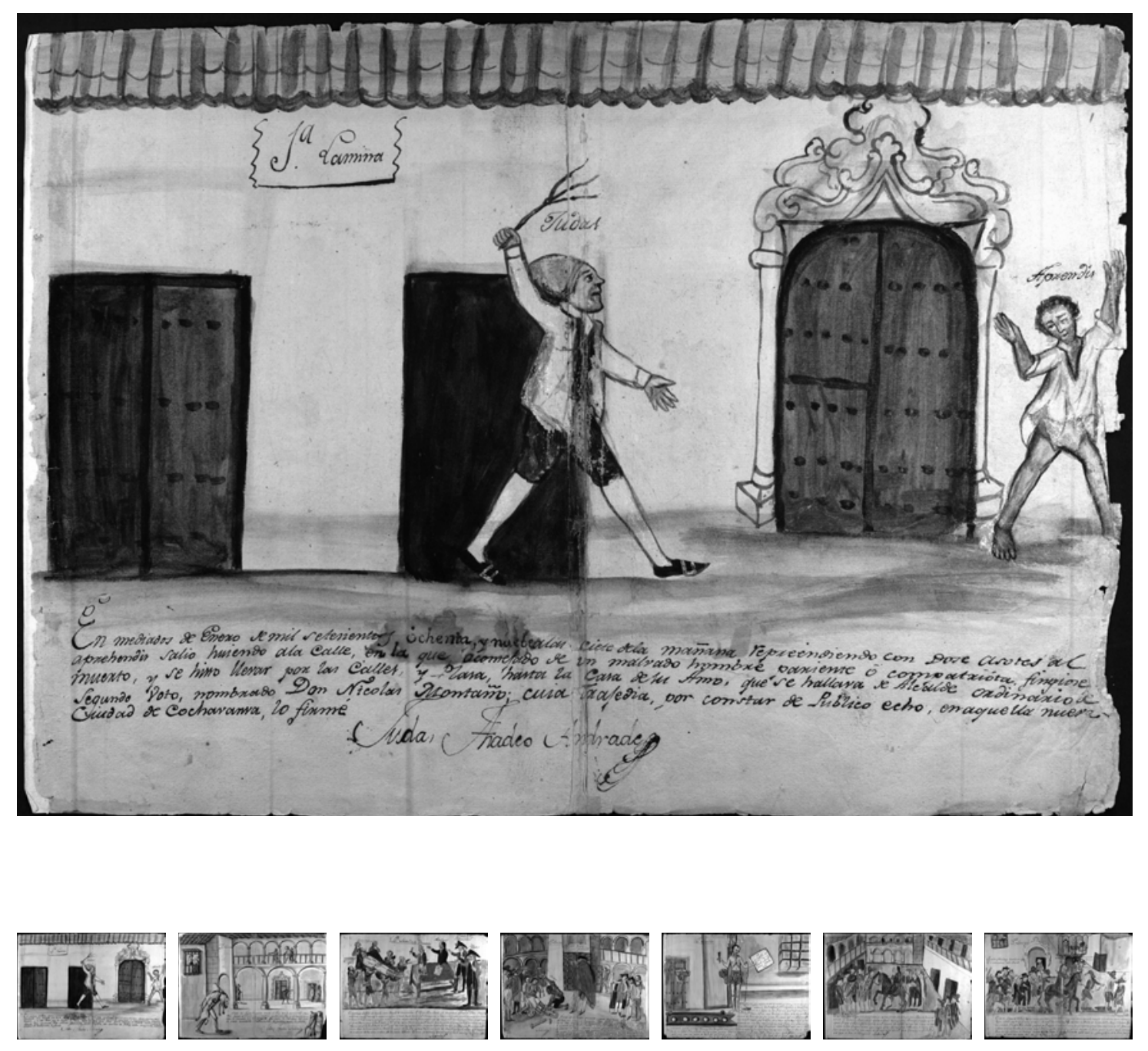

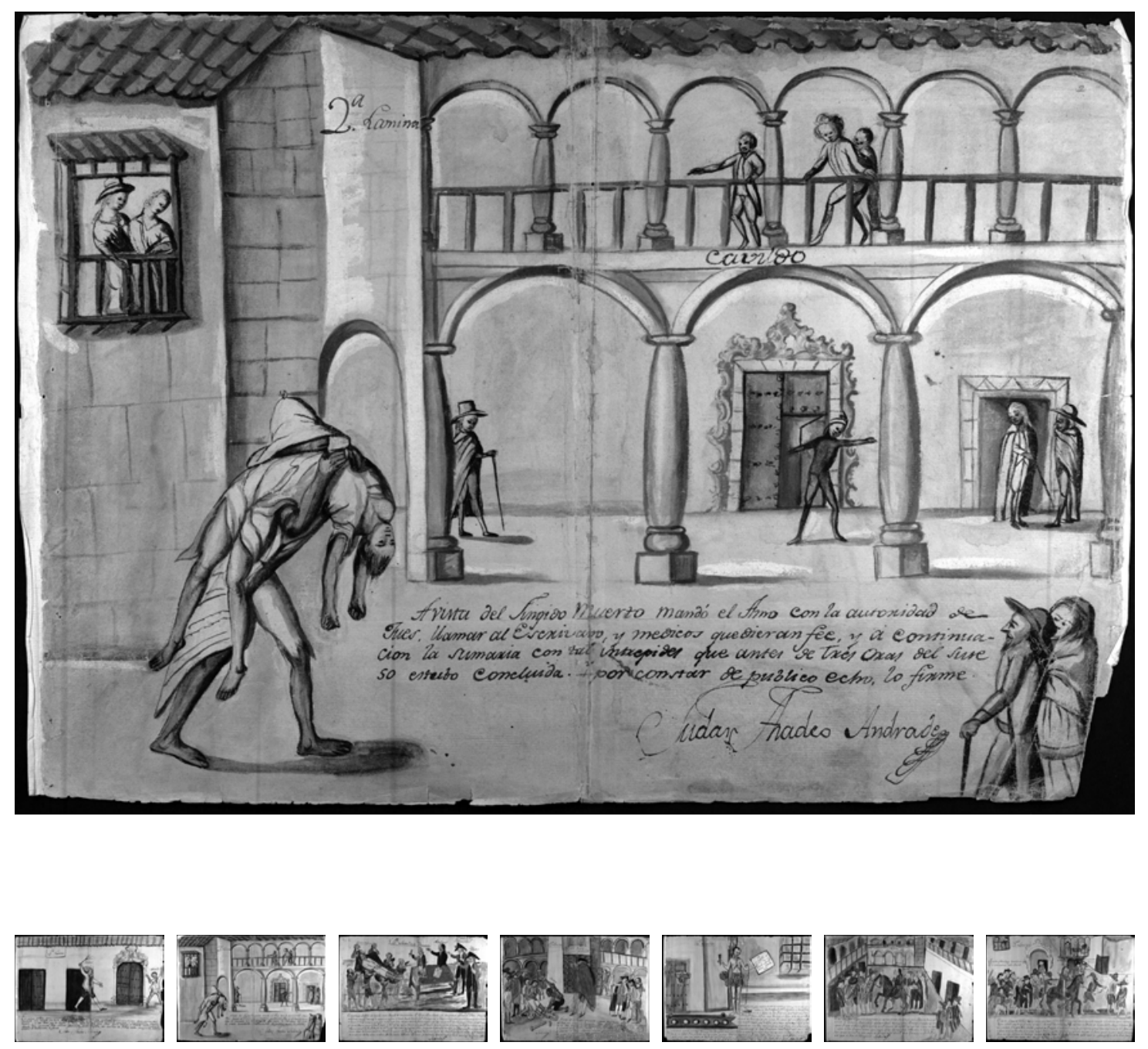

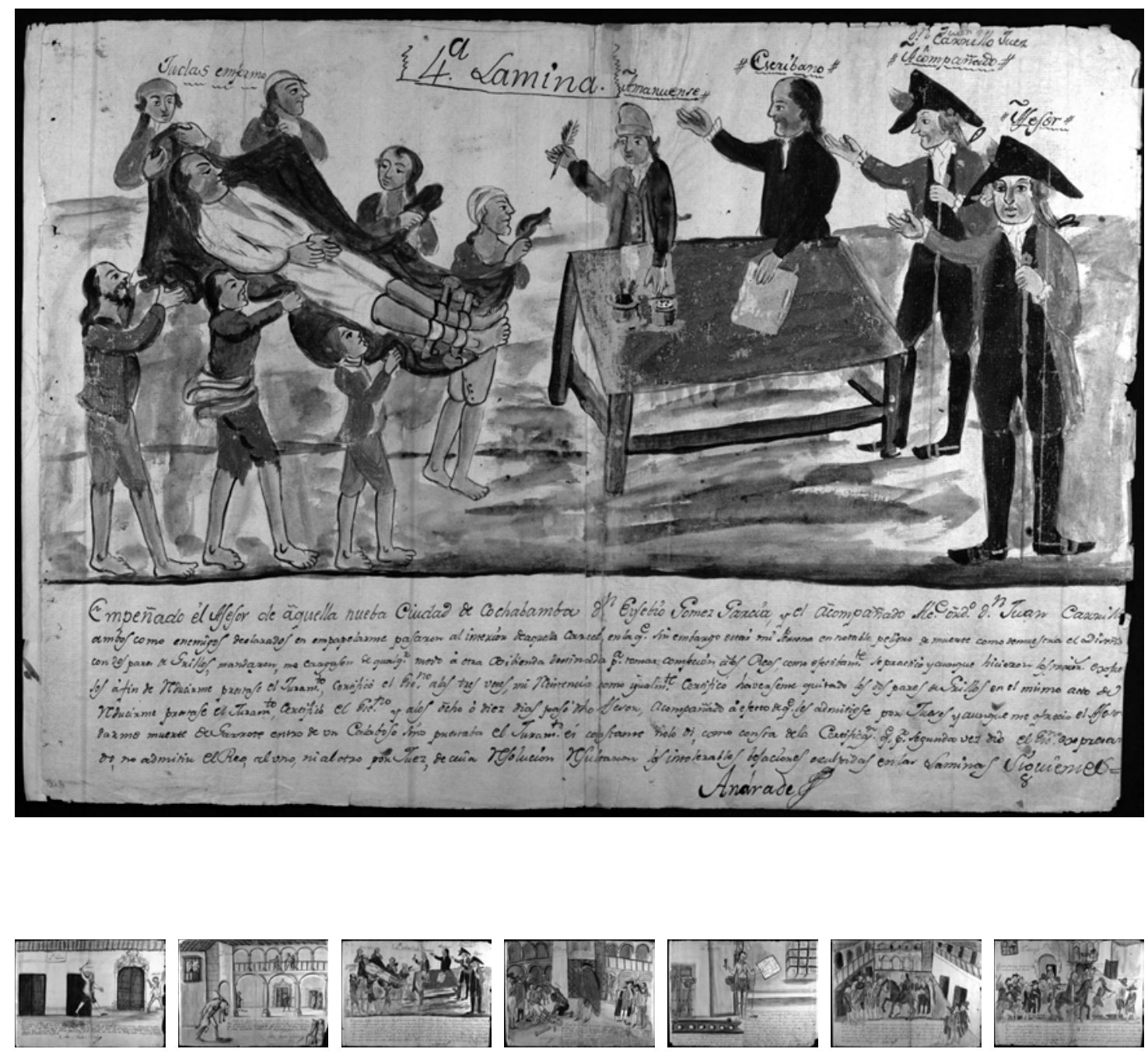

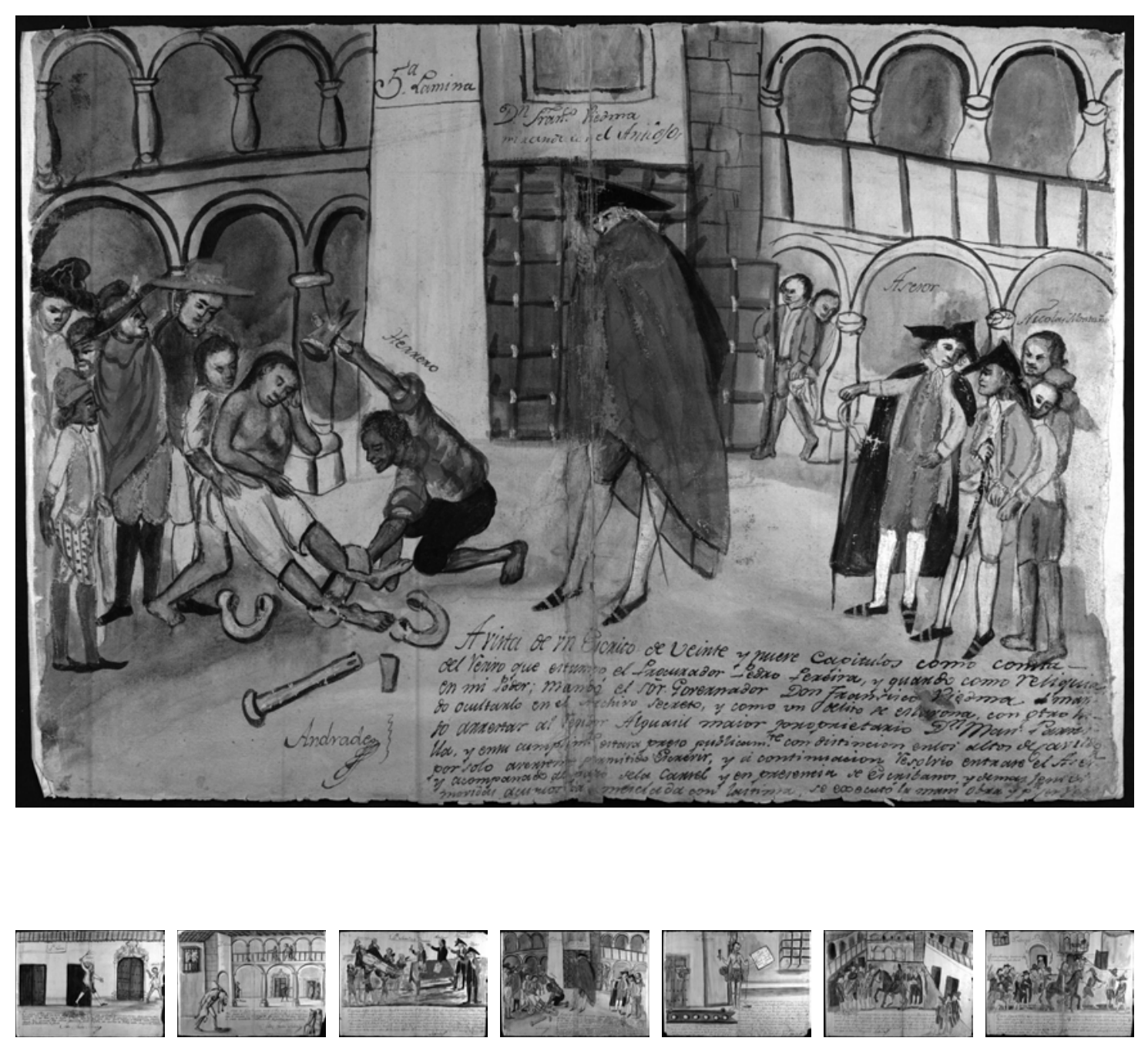

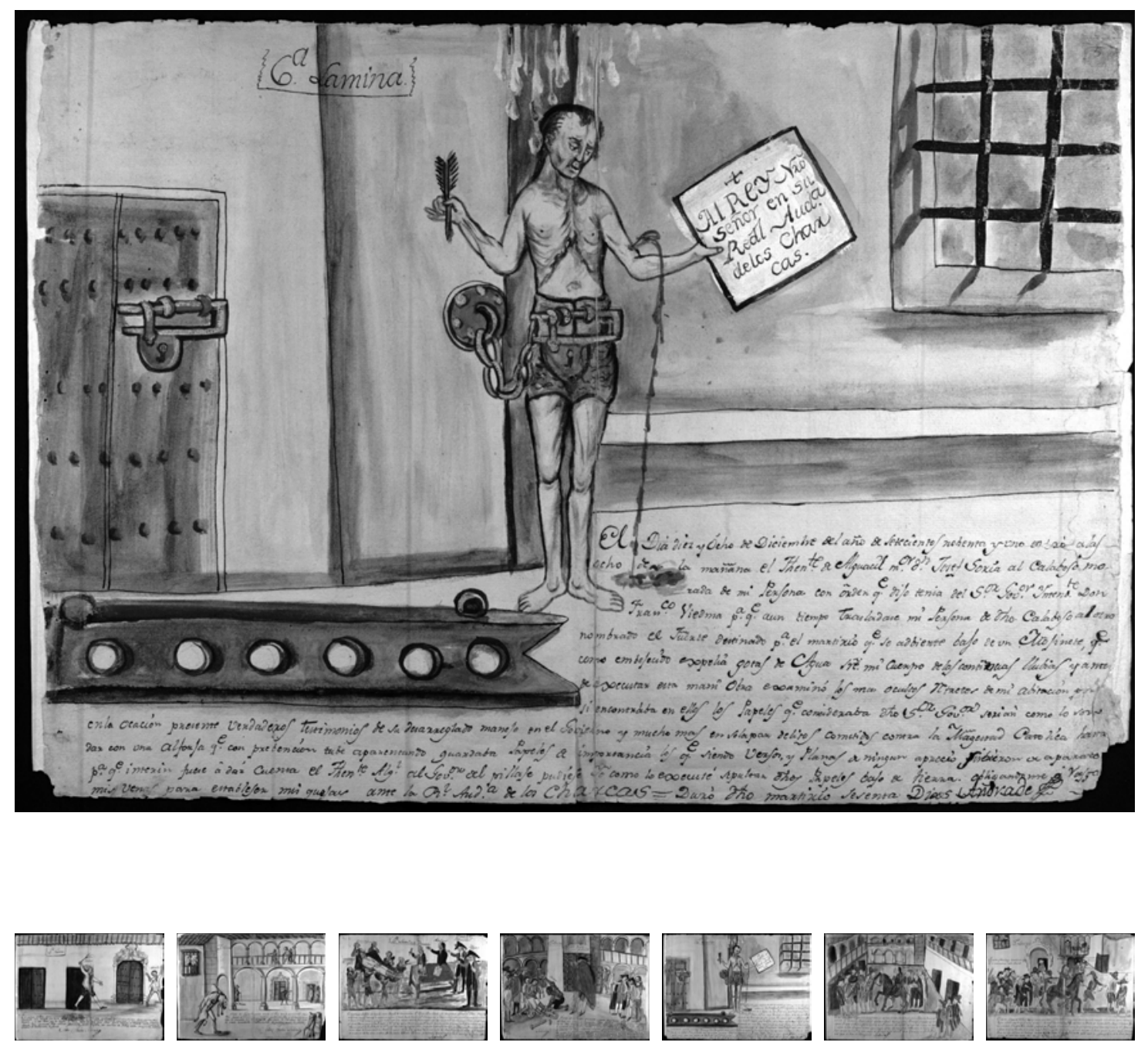

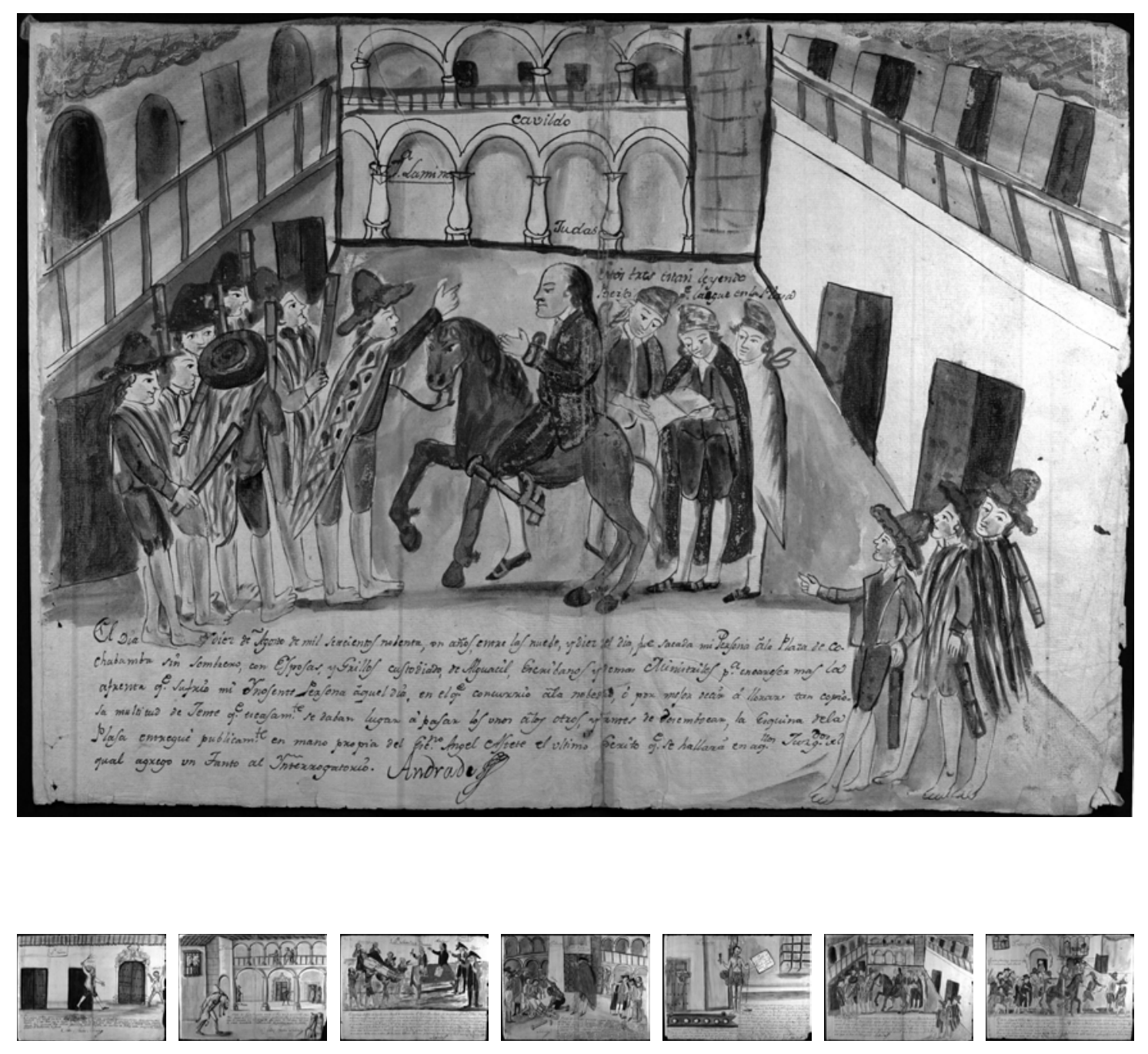

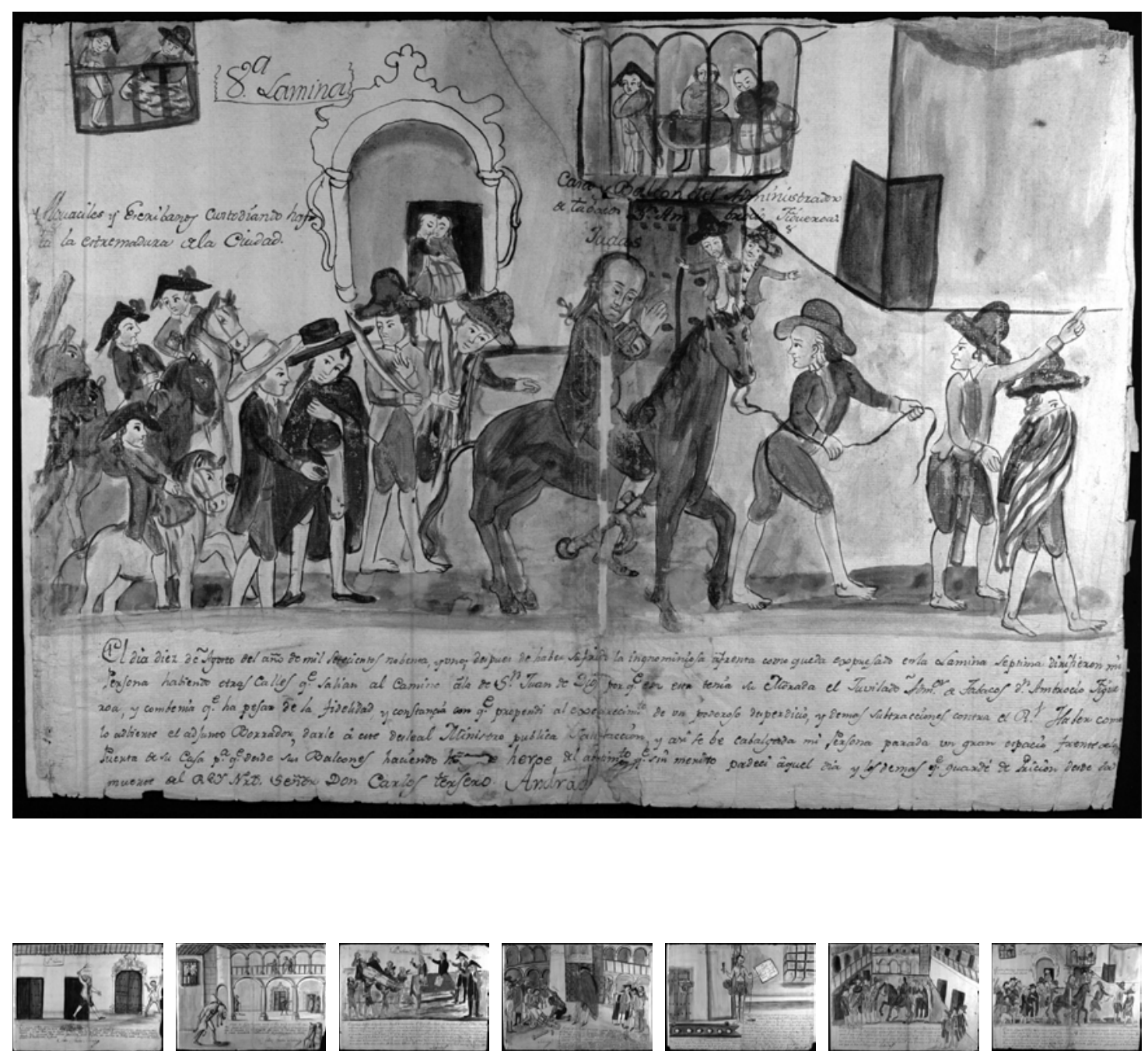